\title{
La desigualdad: un tatuaje que nos acompaña
}

The Inequality: a tattoo that accompanies us

\author{
Gustavo Gordillo de Anda
}

Journal of Economic Literature JEL:

D63, I32, I3

Palabras clave:

Desigualdad, Análisis

de la Pobreza, Bienestar

y Pobreza

Keywords:

Inequality, Analysis of Poverty, Weltare and Poverty

\section{Resumen}

Se analizan las características de la desigualdad en América Latina y se muestra que es un fenómeno generalizado y persistente que invade amplios ámbitos de las sociedades latinoamericanas y no solamente su dimensión económica. Se destacan los mecanismos de trasmisión; se enfatiza en el concepto de heterogeneidad estructural y se advierte que es imposible revisar las políticas económicas en general y más específicamente su carácter o no redistributivo sin considerar las dimensiones políticas, pues su correlación de fuerzas es determinante.

\section{Abstract}

The characteristics of the inequality are analyzed in Latin America and it appears that it is a widespread and persistent phenomenon that invades wide areas of the Latin-American companies and not only his economic dimension. The mechanisms of transmission are outlined; one emphasizes the concept of structural heterogeneity and one warns that it is impossible to check the economic policies more specifically his character or not redistributive without considering the political dimensions, since his correlation of forces is determinant.

\section{Introducción}

Se compara la desigualdad en América Latina mediante la revisión de varios reportes del $\stackrel{\infty}{\sim}$ Banco Mundial, del Programa de las Naciones Unidas para el Desarrollo (PNUD) y de la Comisión Económica para América Latina y el Caribe (CEPAL) y se muestra que en todos ellos se concluye que la desigualdad es un fenómeno generalizado, persistente y que invade amplios ámbitos de las sociedades latinoamericanas y no solamente su dimensión económica, pues -como solía decir el novelista argentino Tomás Eloy Martínez- la desigualdad es un tatuaje que nos marca desde siempre en América Latina. Se destacan los mecanismos de trasmisión, desde los procesos económicos hacia los ámbitos de sociabilidad y los mecanismos del poder y de la toma de decisiones; se enfatiza en el concepto de 
heterogeneidad estructural planteado por economistas como Prebisch, Pinto y Fajnzylber y que retoma Fernando Cortés; como lo señalaron recientemente Santiago Levy y Michael Walton -y hace mucho más tiempo Rolando Cordera-, es imposible revisar las políticas económicas en general y más específicamente su carácter o no redistributivo -al menos en México, sin meter en juego las dimensiones políticas-, pues la correlación de fuerzas y las coaliciones que apoyan o resisten determinadas propuestas económicas son determinantes.

\section{Características de la desigualdad en América Latina y sus mecanismos de trasmisión}

En un importante texto del Banco Mundial sobre la desigualdad en América Latina (2004) este organismo señalaba que América Latina sufre de una enorme desigualdad. El país de la región con la menor inequidad en los ingresos sigue siendo más desigual que cualquier país de la Organización de Cooperación y Desarrollo Económico (OCDE) o de Europa Oriental. Se trata, además, de un fenómeno invasor, que caracteriza múltiples aspectos de la vida, como el acceso a la educación, la salud y los servicios públicos; el acceso a la tierra y a otros activos; el funcionamiento de los mercados de crédito y laborales formales, y la participación e influencia políticas. La inequidad también es persistente; en su modalidad moderna, el alto nivel de desigualdad se origina en las instituciones excluyentes que se han perpetuado desde los tiempos coloniales y han sobrevivido a los diferentes regímenes políticos y económicos, desde estrategias intervencionistas y de sustitución de las importaciones hasta políticas más orientadas al mercado. Incluso en la actualidad, aún persisten significativas diferencias raciales y étnicas (2004:1-2). Estos altos niveles de desigualdad tienen varios efectos negativos sobre el nivel de bienestar en las sociedades latinoamericanas.

- En primer lugar impiden que el crecimiento tenga un efecto dinámico en la reducción de la pobreza.

- En segundo lugar, la desigualdad puede ser un freno para el desarrollo, tanto porque se pierden oportunidades de inversión o de un uso más eficiente de recursos humanos, como porque se incrementan los conflictos distributivos y se pueden erosionar las bases institucionales que de otra forma promoverían el desarrollo.

- En tercer lugar existe un generalizado rechazo al alto nivel de desigualdad por parte de los ciudadanos latinoamericanos como lo muestran, por ejemplo, las encuestas de Latinobarómetro (2004-2010).

En el estudio del Banco Mundial también se interroga sobre las raíces históricas de la desigualdad en América Latina. Siguiendo los planteamientos de Acemoglu, Johnson y Robinson (2001, 2002), y de Engerman y Sokoloff $(1997,2000)$ se enfatiza sobre todo el acceso inicial a recursos naturales -factor endowment-, particularmente en los casos de la minería y el azúcar, que generaron instituciones para administrar el trabajo subordinado - las distintas formas de esclavitud de la población indígena y de la población negra- y la explotación de la tierra desde una visión extractiva. 
En conjunto se afirma en este estudio que:

en términos económicos, los altos niveles de desigualdad se reproducen debido a las interacciones de distintos factores: niveles moderados de desigualdad en la educación (exacerbados por la calidad educacional baja y dispareja); alta rentabilidad de la educación en el mercado, especialmente de la educación superior; altos niveles de concentración de la tierra y otros activos productivos; acceso desigual a mercados clave (en particular, mercados financieros y laborales) y una débil redistribución a través del Estado. De ahí que se propone avanzar en tres ámbitos relacionados con desigualdades en los activos; las instituciones que definen el acceso al mercado y las rentabilidades; y el uso del potencial redistributivo del Estado (2004:11).

El informe del Banco Mundial también se detiene en los efectos sociales de las crisis económicas:

Un hallazgo notable de este informe es la regresividad de las crisis financieras. Las crisis son regresivas porque aquellos más adinerados y mejor informados son los primeros en retirar el dinero de sus países, con lo que a veces disfrutan de las ganancias de capital en términos del poder adquisitivo a nivel local. Las crisis también son regresivas debido a los costos fiscales de los mecanismos de solución que se han adoptado para lidiar con los recientes problemas de la banca en América Latina (2004-16).

Un indicador de lo anterior lo proporciona este informe al señalar que "durante los años ochenta y noventa, las transferencias públicas a grandes depositantes y accionistas de bancos, aplicadas como mecanismos de solución de crisis (es decir, pago de fianzas), se encontraban dentro del margen de 13 a 55\% del Producto Interno Bruto (PIB)" (énfasis mío, ibid).

Esta desviación de recursos hacia sectores de mayores ingresos, junto con los recortes presupuestales que afectaron aún más -como veremos más adelante- a las clases medias y bajas, agravaron la desigualdad. Peor aún como indica el reporte del Banco Mundial "Es poco probable que esas transferencias regresivas a grandes deudores y depositantes -e incluso, ocasionalmente, accionistas- en realidad fueran necesarias para evitar el colapso de los sistemas bancarios" (2004:16).

Como el propio informe subraya, resulta de un enorme valor estratégico el establecimiento de mecanismos institucionales capaces de canalizar y dirimir conflictos distributivos que se derivan de las propias crisis:

dado que nunca se evitarán por completo las crisis, es clave establecer mecanismos de resolución de crisis ex ante eficientes y equitativos (particularmente con respecto a posibles problemas financieros), de modo de evitar las transferencias derrochadoras y muy regresivas que han caracterizado la administración de la mayor parte de las crisis bancarias en el pasado (2004: 17).

El último ámbito al que se refiere el informe del Banco Mundial tiene que ver con lo que se denomina "el uso del poder distributivo del Estado" donde resalta que los tres temas clave son: la reforma fiscal, el suministro adecuado de servicios públicos y la reforma y amplia- 
ción del Estado de bienestar truncado. Respecto al primer tema es interesante reproducir el diagnóstico -conocido en muchos otros estudios- sobre los sistemas impositivos en América Latina:

Aunque los problemas metodológicos abundan en el análisis de las incidencias y es poco lo que se puede afirmar con certeza, una presunción razonable es que, dado el bajo nivel de recaudación de impuestos a la renta personal y a la propiedad, la mayor parte de los regímenes tributarios de la región son proporcionales o sólo levemente progresivos o regresivos. Esta situación significa que los pobres, la clase media y los ricos pagan en impuestos casi la misma proporción de sus ingresos ${ }^{1}$ (2004:18).

Quizá el tema más relevante del conjunto de propuestas presentadas en este informe se refiere a lo que llaman la Reforma y ampliación del Estado de bienestar truncado. Este tema lo introducen de la siguiente manera:

Las transferencias desempeñan un papel importante en todas las sociedades por cuanto ayudan a los hogares a enfrentar los riesgos -por ejemplo, aquellos relativos a la salud, el desempleo y la pérdida de las cosechas- $y$ al redistribuir los recursos hacia los pobres. El argumento a favor de las transferencias redistributivas se acentúa con el hecho de que es probable que la transición a sociedades más equitativas mediante la ampliación de la propiedad de los activos tarde tiempo. Sin embargo, la evolución histórica de América Latina ha dado origen a lo que se podría describir como estados de bienestar "truncados", que proporcionan beneficios sólo a los trabajadores formales (2004:20).

Por su lado, el PNUD realizó un amplio reporte sobre el tema de la desigualdad en 2010 bajo el título de Actuar sobre el futuro: romper la trasmisión inter-generacional de la desigualdad. El informe está organizado en 6 capítulos. El primero se refiere al enfoque del Informe fuertemente anclado en el marco conceptual que ha propuesto y desarrollado el economista hindú Amartya Sen (1980, 1985, 1998). Crucial es el uso de los conceptos de capacidades ${ }^{2}$ y funcionamientos. Según este enfoque, el desarrollo es concebido como una expansión de la libertad (Sen, 1999) y la escala del conjunto de opciones de vida -posibilidades de "ser" y "hacer"- disponibles para un individuo es la medida de su libertad efectiva. En este marco conceptual, las capacidades se refieren precisamente al conjunto de opciones entre las cuales las personas pueden elegir, y los funcionamientos son los elementos específicos contenidos en dicho conjunto que materializan las capaci-

1 Ver figura 1.7 capítulo 9 del estudio del Banco Mundial, 2004.

2 Como señalan los autores del Informe: "Existe un debate sobre el uso del término capacidades como traducción de capabilities, el concepto original de Sen (1980; 1985). En la teoría de Sen, capability se refiere a capacity (capacidad de hacer algo) más ability (poder hacer bien determinada actividad). Algunas traducciones al español optan por la expresión capacidades humanas. Otras, siguiendo el ejemplo del inglés, utilizan el término capabilidades, inexistente en español, que surge de la fusión de capacidades y habilidades. En este Informe, para simplificar, se opta por emplear el concepto capacidades. Las itálicas indican que esta palabra es utilizada como un término teórico que no sólo hace referencia a la idea de poder realizar una actividad, propia del término "capacidad", sino que también se refiere a la noción de destreza adquirida, propia del concepto de "habilidad" (Nebel y Flores-Crespo, 2010)" $(2010,15)$. 
dades al transformar el acceso a bienes y servicios en aquello que una persona efectivamente logra hacer o ser en distintas etapas de su vida ${ }^{3}$ (2010:17). Pero el mensaje central del Informe está basado en las siguientes premisas:

[...] 2) La desigualdad en ingresos, educación, salud y otros indicadores persiste de una generación a otra y se presenta en un contexto de baja movilidad socioeconómica. (2010-19) 3) Lo anterior permite afirmar que existe un mecanismo de reproducción del logro que se transmite de una generación a la siguiente [...]

4) La desigualdad como la pobreza son fenómenos causados por restricciones. [...]En concreto, se observa que la determinación de las aspiraciones y la percepción que los miembros de un hogar tienen acerca de la posibilidad de alcanzar los objetivos que se han planteado están vinculadas con, e intensamente influenciadas por, las restricciones del contexto y los marcos de referencia en que esas personas se desenvuelven.

5) [...] Existen otros factores que impiden que las políticas públicas compensen las brechas y la heterogeneidad del logro en bienestar [...] Entre estos factores destacan la baja calidad de la representación política, la debilidad de las instituciones, el acceso diferenciado a la influencia sobre el diseño y la aplicación de políticas específicas, los problemas clásicos de la acción colectiva y las fallas institucionales que derivan en corrupción y captura del Estado. [..] 6) Las políticas públicas más extendidas en la región se han enfocado, con éxito en muchos casos, en aspectos específicos del combate a la pobreza [...] Un enfoque como el que propone este Informe, que analiza la desigualdad en términos de capacidades y de la equidad en la expansión de la libertad efectiva de los hogares, implica una perspectiva más amplia, que analiza de manera concreta las restricciones objetivas y subjetivas que enfrentan, de manera más directa y acuciante, los grupos más vulnerables de ALC (2010-20).

Los autores del Informe basados en el trabajo de Genicot y Ray (2009) utilizan el término de ventanas de aspiraciones que consiste en un espectro de posibilidades para la elaboración de aspiraciones, abiertas como resultado de las interacciones con la red social más cercana -de pertenencia-y otras informaciones -como la derivada de los medios de comunicación-. Añade a lo anterior el término de brecha de aspiraciones que como su nombre lo indica sería la distancia entre lo que una persona es o tiene en el presente y lo que espera alcanzar. Esta brecha de aspiraciones puede conducir, según los autores, a tres situaciones. Si la distancia que expresa la brecha es razonable habrá incentivos para cerrarla. Si por el contrario la brecha es muy grande habrá poco incentivos para que se

3 Una perspectiva alternativa para analizar la desigualdad es el Enfoque de la Cooperación para el Desarrollo basado en los Derechos Humanos, denominado comúnmente enfoque de derechos. En el recuadro 1.1 basado en OHCHR (2006) y Yasukawa, Ortuño y Brockmann (2009), el informe del PNUD rinde cuenta de este enfoque alternativo: El enfoque de derechos, tal como lo comprende el sistema de las Naciones Unidas según la Declaración de Entendimiento Común sobre el enfoque basado en derechos de 2003, establece una serie de elementos básicos que deben guiar el diseño de los programas de cooperación y desarrollo. Las políticas deben tener por objetivo principal la realización de los derechos humanos y el fortalecimiento tanto de las capacidades de los titulares de obligaciones para cumplir con sus deberes como de las capacidades de los titulares de derechos para reclamar sus derechos. Asimismo, los estándares de derechos incluidos en los instrumentos internacionales de derechos humanos deben orientar la cooperación y las políticas para el desarrollo en todas las fases del proceso de programación (2010-18). 
reduzca o cierre. Finalmente. "existe la posibilidad de que el esfuerzo se vea inhibido por la ausencia de alternativas en el entorno de las personas" (2010-84).

Las dos últimas situaciones se refieren a sociedades con alta polarización o como las llaman en el Informe, "sociedades desconectadas":

Una sociedad donde la distribución del ingreso está conectada en todas partes, de tal manera que en la vecindad del nivel de ingresos de cada individuo u hogar existan siempre otros niveles de ingresos que estén relativamente cercanos, generará ventanas de aspiraciones más amplias, con al menos un tramo ascendente asequible para cada nivel de esfuerzo.

El capítulo 5 del Informe denominado "Del hogar a la polis: la economía política de la persistencia de la desigualdad" intenta brindar una explicación de conjunto sobre la persistencia y reproducción de la desigualdad en América Latina. Parte de lo que denomina: La trampa sistémica de la desigualdad:

Este capítulo se centra en el análisis de los factores que contribuyen a la persistencia de la desigualdad en el ámbito de los procesos sistémicos, los cuales se localizan en el sistema político y en el Estado, y afectan principalmente las formas que asume el proceso de representación política. El objetivo es explicar cómo los procesos de toma de decisiones en la elaboración y la implementación de políticas públicas y las formas en que se agregan los intereses de los distintos grupos pueden contribuir a la persistencia de la desigualdad o dificultar en gran medida su reducción (2010:95).

Los dos ejemplos que utilizan para ilustrar esta argumentación son por una parte la reforma fiscal -o más bien su restringida implantación- y por otra parte la capacidad regulatoria del Estado (2010: 95-6). Respecto a la reforma fiscal el cuadro 5.1 (p. 96) expresa bien la baja capacidad recaudatoria de los estados latinoamericanos en general -como porcentaje del PIB en 2005- en comparación con otras regiones del mundo y su fuerte dependencia de los impuesto al consumo o indirectos. Respecto a la capacidad regulatoria del Estado, el Informe plantea que:

resulta claro que los grupos más vulnerables no sólo ven limitado su acceso a los bienes y servicios disponibles, sino que también tienen menos posibilidades de hacer escuchar su voz en su calidad de consumidores [...] Algunos estudios destacan que las empresas con poder de mercado suelen tener gran capacidad de influencia en el sistema político y legal, lo cual les permite protegerse de las acciones regulatorias del Estado (ver, por ejemplo, Guerrero, López-Calva y Walton, 2009) (2010: 96).

En este caso el argumento es que el "poder regresivo del mercado" refuerza la persistencia de la desigualdad en la distribución de ingresos entre hogares y entre regiones. La argumentación sobre la forma en que esas tres variables interactúan se resume en el siguiente argumento: 
El argumento se basa, en primer lugar, en las condiciones iniciales, caracterizadas por altos niveles de desigualdad y debilidad institucional del Estado. El segundo elemento son los mecanismos de transmisión: i) el acceso diferencial a la influencia sobre las políticas públicas (que incluye los problemas de calidad de la representación, la debilidad de los mecanismos de rendición de cuentas y la captura del Estado), y ii) la existencia de mercados que funcionan de manera ineficiente, fundamentalmente porque existen estructuras de mercado concentradas y algunas empresas ejercen poder dominante sobre los mercados (2010:96).

En consecuencia una eficaz redistribución de los ingresos requiere rendición de cuentas y ejercicio efectivo de la competencia política, o como lo planteó otro Informe del PNUD sobre la democracia en América Latina (2004) una democracia de calidad con una ciudadanía de alta intensidad.

En síntesis:

la reducción de la desigualdad debe constituir en sí misma un objetivo central de la política pública. Por las razones normativas y prácticas expuestas en este Informe, la desigualdad debe ser combatida mediante los instrumentos explícitos propios de las intervenciones públicas que están en condiciones de lograr su reducción. Este objetivo debe ser concebido como el complemento de una política integral de protección social y de provisión de servicios de calidad con componentes universales (énfasis mío, 2010: 110);

para después retomar el planteamiento de Amartya Sen (2005):

lo que denominó el análisis de "Triple R", el cual podría traducirse como el análisis de Alcance (Reach), Amplitud (Range) y Apropiación (Reason). En términos simples, este análisis implica, en primer lugar, que las acciones públicas deben llegar a las personas, los hogares y las comunidades para quienes fueron diseñadas (alcance). Luego, para ser eficaces, estas intervenciones deben ser comprensivas, es decir, deben atacar el conjunto de las restricciones operativas identificadas (amplitud). Finalmente, estas acciones deben ser consistentes e incidir sobre las aspiraciones, los objetivos y la autonomía de los beneficiarios, promoviendo que estos se conviertan en sujetos y no en receptores pasivos de las políticas de desarrollo (apropiación) (2010:115-16).

La relevancia de los tres textos producido por agencias multilaterales con gran influencia en la elaboración y la implementación de políticas públicas, no puede ser subestimado. Lo que sorprende aún más que la convergencia de visiones sobre la desigualdad en América Latina es la similitud con opiniones y visiones que datan de las mejores épocas de CEPAL con Prebisch ${ }^{4}$ a la cabeza y luego con pensadores como Aníbal Pinto ${ }^{5}$ y Fernando Fajnzylber. $^{6}$

4 El texto clásico de Prebisch es El desarrollo económico de la América Latina y algunos de sus principales problemas, 1949. En CEPAL, 1998.

5 Heterogeneidad estructural y modelo de desarrollo reciente de la América Latina en Inflación: raíces estructurales, 1973.

6 Industrialización en América Latina: De la caja negra al casillero vacio, 1989. 
Fernando Cortés en incisivo ensayo intitulado Desigualdad económica y poder (2011) comienza por reivindicar elementos centrales de estos tres economistas en relación con el vínculo entre crecimiento y desigualdad:

Los primeros planteamientos de la CEPAL sobre las condicionantes de la desigualdad en la distribución del ingreso se remontan a Prebisch (1949), quien en disputa con las teorías dualistas dominantes en aquella época (Lewis, 1960), sostuvo que el subdesarrollo era un modo de funcionamiento y no un simple atraso (lo que abría también un frente con la teorías que suponían que el crecimiento seguía etapas férreas; etapas que serían sistematizadas años después por Rostow en 1960).

Como lo subraya Cortés, el concepto clave en este corriente de pensamiento es el de heterogeneidad estructural que deriva de la forma en que con sectores exportadores de materias primas los países latinoamericanos tienden a enormes disparidades entre los mercados laborales:

El concepto heterogeneidad estructural acuñado por Prebisch alude a la dispersión del desarrollo tecnológico en las economías periféricas que conlleva un amplio abanico de la productividad del trabajo y por tanto a una desigual distribución del ingreso. Este argumento inscribe la desigualdad en un modelo económico que de persistir la especialización productiva [...] explotando las ventajas comparativas sumerge a los países de América Latina en una trampa de subdesarrollo (2011-2)

Aníbal Pinto en los setentas precisa más este concepto señalando que la heterogeneidad estructural tiene su origen en las economías de enclave -sean explotaciones mineras o plantaciones.

La siguiente etapa en el refinamiento de este concepto de heterogeneidad estructural es dado según Cortés en los ochentas por el Programa Regional de empleo para América Latina y el Caribe (PREALC):

El planteamiento de PREALC sostenía que el sector informal urbano en la región se generaba por el crecimiento natural de la población en las ciudades, por una parte, y por las migraciones rurales urbanas. [...] El sector informal urbano se caracterizaría (i) por la utilización de tecnologías simples y mano de obra con bajos niveles de calificación y (ii) porque sus actividades se desarrollan en mercados altamente competitivos (Tokman 1979: 76 y 77) (2011: 3).

Por su parte Fajnzylber (1989) reflexionaba sobre las posibilidades de desencadenar un proceso de desarrollo autosostenido a base de una industrialización impulsada por el Estado pero partiendo de una transformación estructural del campo. Como señala Cortés, Fajnzylber reflexiona sobre las relaciones entre la estructura del poder y la distribución del ingreso: 
En ciertos países la élite rentista influye en algún grado en perjuicio de la equidad, tanto directamente, por la concentración de la propiedad, como en forma difusa por la existencia de una institucionalidad y de políticas económicas que tienden a consolidar un sistema de distribución de los beneficios del progreso coherente con la distribución primitiva del poder. Una apertura radical del mercado interno podría poner en tela de juicio o racionalizar el sistema industrial rentista, pero si no se modifica la distribución primitiva del poder y éste se concentra, el retroceso industrial consiguiente hará que se acentúe la integración en el mercado internacional a base de los recursos naturales y del desarrollo de intermediación comercial y financiera (1989: 62 citado por Cortés 2011-4).

Cortés da cuenta de recientes desarrollos en el pensamiento económico que analizan la desigualdad desde las imperfecciones del mercado. En efecto, se refiere a un libro reciente de Santiago Levy -el principal inspirador desde el sector público del programa Oportunidades - y Michael Walton (2009) y sobre todo un artículo, elaborado en el thinktank de Washington D. C., el Center for Global Development, por Nancy Birdsall -ex alta funcionaria del BID-, Augusto de la Torre -economista principal del Banco Mundial para América Latina y el Caribe- y Felipe Valencia (2010). En éste último se señala que:

altos niveles de desigualdad no sólo dificultan el crecimiento y la reducción de la pobreza sino pueden contribuir a disminuir el crecimiento lo cual, a su vez, hace difícil reducir la desigualdad. Instituciones y políticas, en contextos con altas tasas de pobreza y marcada concentración del ingreso pueden ser fuentes de ineficiencias y crecimiento exiguo (2010:31 citado por Cortés, 2011:9).

Un análisis del caso mexicano realizado bajo esta perspectiva concluye que la desigual distribución del poder, expresada en la riqueza extrema y el control operativo en el sector empresarial, por un lado, y en los sindicatos heredados de la época del corporativismo por otro, se han constituido en condicionantes de la desigualdad y obstáculos para el desarrollo de México, impidiendo el diseño de políticas y el funcionamiento de las instituciones que lesionan sus intereses (Guerrero, López y Walton, 2009: 112 y 152; citado por Cortés 2011: 9).

Por su lado sus autores señalan que el informe del PNUD:

procura, de manera concreta, orientar el debate público para poner a la desigualdad en el centro de las prioridades de las políticas públicas y proponer medidas específicas que favorezcan su reducción y, por tanto, una mayor eficacia en el combate a la pobreza, un crecimiento económico acompañado de mayor inclusión, una mayor eficacia en el funcionamiento del Estado y una mejor calidad de los sistemas de representación política (2010:117).

En consecuencia no parece que el papel del Estado en el desarrollo o para enfrentar específicamente la desigualdad sea una diferencia sustancial entre las dos corrientes de pensamiento a las que se refiere en su texto Fernando Cortés. Al final parece más bien que la discusión, en la medida que se adentra en el eje crucial de los derechos sociales universales, vuelve a colocar en el centro del debate cómo financiar un estilo de desarrollo 
que tenga en el centro el combate a las desigualdades. $Y$ en esto aunque no lo agote, la reforma fiscal adquiere primacía.

Retomando el tema de la persistencia de la desigualdad, Cortés a partir de diversos autores aportan elementos interesantes de reflexión:

Resultados de investigaciones empíricas muestran que la desigualdad en la distribución del ingreso: (i) no difiere significativamente entre países democráticos y autoritarios y cuando sí se registran diferencias éstas suelen no ser robustas (Przeworski, 2010: 147-49), (ii) es bastante estable en períodos cortos ( $\mathrm{Li}$, Squire y Zou, 1997: 1-18) y los aumentos suelen ser más rápidos que sus declinaciones, tal como muestra la experiencia de los países del ex-bloque soviético después de 1989 y la lenta pero persistente caída de la desigualdad en México registrada desde 1950 a 1984, su alza brusca entre 1984 y 1989 y su relativa estabilidad desde ese último año hasta el presente (Cortés, 2008).

\section{La desigualdad en México, sus tendencias}

Ahora me interesa señalar algunos aspectos relacionados con la desigualdad en los últimos 20-25 años en México. Para ello contrastaré las visiones de Nora Lustig et al (2010) con la visión de Fernando Cortés $(2011,2008)$ y luego retomaré algunas de las reflexiones de largo alcance que propone Rolando Cordera $(2006,2008)$.

En un capítulo de un libro editado por Lustig y López (2010), Esquivel, Lustig y Scott (2010: 175-217) argumentan que justo en el período 2000-2006 en que el PIB per cápita cayó a uno por ciento anual -de un ritmo promedio anual de 4\% entre 1996-2000-, es cuando la desigualdad en el ingreso comenzó a reducirse más. Examinan en su texto hasta dónde esta reducción en la desigualdad es producto de una declinación en el premio al trabajo asalariado calificado y analizan los cambios en la composición de la fuerza de trabajo en términos de educación y experiencia al tiempo que buscan establecer una conexión entre esa composición y cambios en el gasto público. En particular los autores analizan la contribución de PROGRESA/OPORTUNIDADES en la reducción de la desigualdad en el ingreso no salarial (2010:176). La figura 7.1 de este texto (2010:177) muestra la evolución del coeficiente de Gini desde 1984 hasta 2006. Se perfila como una U invertida en donde alcanza su cúspide a mediados de los noventas, pero desde 1996 y sobre todo 2000 tanto el coeficiente de Gini para ingreso total de los hogares como el coeficiente de ingresos monetarios se reducen de 0.543 a 0.498 y de 0.539 a 0.506 respectivamente.

Utilizando otra forma de medida, a través de las curvas de incidencia del crecimiento (GIC, según sus siglas en inglés) sugerida por Ravallion y Chen (2003), Esquivel (2008) demuestra como el mayor efecto de este crecimiento en los ingresos ocurre en las localidades rurales -definidas como localidades de menos de 15 mil habitantes- y consecuentemente y dada la brecha de ingresos entre localidades urbanas y rurales, tiene un efecto de reducción de la desigualdad. Lo que ocurre por tanto es un incremento mayor del promedio entre los deciles de ingreso más bajo y un incremento abajo del promedio en el decil de mayores ingresos (2010:178-80). Estos resultados sugieren según los autores, una serie de factores que interactuaron en este periodo 1996-2006 y sobre todo entre 2000-2006. Dichos determinantes próximos - proximate determinants- son: la proporción 
de adultos entre el total de miembros de la familia, de adultos trabajadores en el total de adultos de la familia, los ingresos laborales por adulto trabajador en la familia, y el ingreso no salarial por adulto que incluye transferencias públicas y remesas. Los autores señalan que el cambio más dramático ocurrió en el rubro de los ingresos no salariales:

En 1996-2000 los cambios en el ingreso no salarial contribuyeron con un magro 0.4 por ciento en la reducción de la desigualdad. En contraste en 2000-2006 contribuyeron con un15.1 por ciento a la reducción de la desigualdad, haciendo del ingreso no-salarial el segundo más importante contribuyente a eso.

Pero ingreso no-salarial es un concepto heterogéneo. Incluye ingreso derivado del capital (como utilidades, intereses y rentas) que tiende a concentrarse en la parte alta de la distribución del ingreso. Pero también incluye transferencias privadas (como las remesas) que tiende a estar más concentrada en los rangos medios y medios bajos de la distribución del ingreso. Finalmente ingreso no-salarial también incluye transferencias gubernamentales (como pensiones) que están concentradas en los rangos medios y medios superiores del ingreso, así como transferencias gubernamentales focalizadas (como el programa de transferencias condicionales en efectivo Progresa/Oportunidades) que se concentran en la parte baja de la distribución del ingreso (traducción directa del inglés, 2010: 182-83).

Tres factores socio-demográficos deben ser subrayados como lo hace Esquivel (2008): la reducción en el tamaño promedio del hogar -de 5.68 en 1996 a 4.97 en 2006, el incremento de los adultos trabajadores de $58 \%$ en 1996 a $62 \%$ en 2006 , cambios que implican una reducción de la tasa de fertilidad y un incremento en la participación femenina en la fuerza de trabajo, ambas tendencias que impactan más en los sectores más pobres de la población (2010:183). El efecto combinado de ambos, como se confirma en el Censo de población 2010, es una reducción de la tasa de dependencia.

Esquivel, Lustig y Scott pasan en seguida a analizar las fuentes de ingreso y encuentra tres que aumentan las desigualdades y tres que las disminuyen. Las primeras son ingresos que provienen de empresas propias (ganancias), ingreso derivado de la propiedad (rentas) y pensiones. El impacto de las tres se incrementó entre 1994-2006, siendo importante el rubro de pensiones en este respecto. Las tres fuentes de ingreso que reducen la desigualdad son los ingresos salariales, las remesas y las transferencias públicas:

Las remesas tuvieron un efecto nacional porque estuvieron fuertemente concentradas en la mitad de abajo de la distribución del ingreso. Redujeron la desigualdad del ingreso al reducir la brecha urbano-rural y no por reducir la desigualdad al interior de cada uno de estos sectores. Lo anterior cambió en 2000 y más decisivamente en 2006... Las transferencias públicas redujeron la desigualdad tanto en el ámbito nacional como en el sector urbano y en el sector rural. Su efecto creció con el tiempo. Para 2006 las transferencias se convirtieron en la fuente de ingreso con el efecto más amplio en la reducción de la desigualdad...por tres razones: su participación en el total de ingreso aumentó, la desigualdad en la distribución de las transferencias se redujo y su correlación del coeficiente de Gini con el ingreso total monetario cayó (2010: 186). 
Pero la parte más importante en términos de explicar la reducción de la desigualdad entre 2000-2006 tiene que ver con la evolución en la desigualdad del ingreso salarial, se entiende el ingreso salarial como el resultado de multiplicar las horas trabajadas por los salarios horarios incluyendo las remuneraciones a los auto-empleados. Suponiendo que las horas trabajadas no cambiaron mucho en este periodo de análisis, indican los autores, el cambio en la desigualdad de los ingresos salariales debió provenir de cambios en el ingreso salarial. Los autores se centran en analizar una dimensión de esta desigualdad: la brecha entre salarios de trabajadores calificados y no calificados.

Los autores hacen suya las conclusiones del análisis de Esquivel (2008) respecto a los factores que aumentaron la desigualdad entre los ingresos de los trabajadores calificados y los no calificados y concluyen que esa desigualdad es producto más que de una reducción relativa de los salarios de los no calificados de un incremento en los ingresos de los trabajadores más calificados o con más experiencia (2010: 189-190). Consideran el impacto de dos factores en este resultado. Por un lado un cambio tecnológico sesgado hacia los trabajos más calificados, inducido exógena o endógenamente a través de multinacionales o de empresas exportadoras.

Para el periodo post TLC hay tres explicaciones para la reducción de la desigualdad en el campo de los ingresos salariales: el incremento en la oferta de los trabajadores calificados y el incremento de la demanda de trabajadores no calificados derivado de la ampliación del sector de maquiladoras. La tercera explicación tiene que ver con un premio al trabajo no calificado que llega con retraso tanto porque el impacto de la liberalización comercial en los salarios se tomó unos años antes de manifestarse o porque como se dijo el efecto de un cambio tecnológico sesgado hacia el trabajo calificado (2010-190).

En síntesis, concluye el estudio, "Progresa/Oportunidades es un ejemplo de "eficiencia redistributiva". Con apenas 0.36 del PIB y $4 \%$ del gasto redistributivo, el programa responde por $18 \%$ del cambio en el Gini post transferencia y $81 \%$ del Gini después de la inclusión de programas focalizados en los pobres" (2010:198).

Pero el aspecto más decisivo en términos de políticas públicas es que, como señalan los autores, $58 \%$ del gasto gubernamental redistributivo es regresivo, y de éste el $11 \%$ en realidad aumenta la desigualdad de ingresos (2010:199).

El cuadro 7.3 (2010: 203-04) a partir de un estudio de Scott (2009) presenta una lista de los principales programas gubernamentales redistributivos y el cuadro 7.2 (2010: 201-202) presenta estos programas y su asignación presupuestal en 2006. Estos programas y categorias redistributivas -25 en total- alcanzaron en 2006 cerca de 100 mil millones de dólares, es decir 10\% del PIB nacional, $12.8 \%$ del ingreso primario de los hogares -ingreso prefiscal- y 60\% del gasto público total (2010-202). Desagregado el gasto total redistributivo presentado en el cuadro 7-2, las transferencias en especie para la educación y la salud representan 42 y $26 \%$ del total, respectivamente. El restante $32 \%$ se distribuye de la siguiente manera: $11 \%$ son subsidios generales para la electricidad en zonas residenciales y para gasolina, $9 \%$ para pensiones -la parte subsidiada-, $7 \%$ para subsidios agrícolas y 5\% para transferencias monetarias para los pobres (2010:205).

Utilizando el coeficiente de concentración que es "un coeficiente Gini de la distribución de las transferencias -o impuestos dependiendo del caso- la población clasificada de acuerdo con su ingreso corriente per cápita pre-transferencias", aparece Oportunida- 
des como el programa más progresivo y el programa rural Ingreso-Objetivo como el más regresivo. La mayor parte de los programas focalizados en los pobres son progresivos. Lo más regresivos son las transferencias monetarias a productores rurales y las pensiones subsidiadas a empleados de algunas empresas para-estatales:

En medio hay un gran número de programas y categorías que son regresivas en términos absolutos pero progresivas en términos relativos: por ejemplo subsidios a la gasolina, al gas, a la electricidad residencial y otros subsidios al consumo como la exenciones al IVA, los beneficios del seguro social o la educación media superior y superior." (2010: 206 y cuadro 7-10).

La conclusión general de este texto es que la reducción de la desigualdad que ocurre entre 1996 y 2006 -con un reducción de $1 \%$ anual de 2000 a 2006- y el mejoramiento en ingresos en los deciles de personas más pobres está ligado a tres rasgos: mayores salarios relativos de los trabajadores menos cualificados, un incremento en la proporción de recursos obtenidos a través de las remesas y la expansión de las transferencias gubernamentales a los más pobres (2010-210).

Los mismos autores previenen ante cualquier falso optimismo frente a esta peculiar reducción de la desigualdad en México:

La desigualdad en el ingreso ha estado reduciéndose en un período de bajo crecimiento. Si el bajo crecimiento es una consecuencia del hecho que no están siendo creados muchos empleos en los sectores de alta productividad y a su vez es una consecuencia de los altos costos que enfrentan las empresas en sector formal; entonces tanto el bajo crecimiento y la caída en la desigualdad de ingresos es el resultado de un mismo proceso indeseable. Levy (2008) argumenta que eso es exactamente lo que ha pasado en México y el mayor responsable, en algunos aspectos es la propia política social que ha creado desincentivos para la generación de empleos en el sector formal. Pages, Pierre y Scarpetta (2009) encuentran evidencias que la creación de empleos en México -y otros países latinoamericanos- se dio de manera desproporcionada en los sectores de baja productividad y bajos salarios. A eso le llaman "empleos sin crecimiento" (growthless jobs). (2010: 212 pie de página 101)

Por su lado, Fernando Cortés (2011) concluye en cambio que "como consecuencia de la apertura de la economía, el aumento de la inversión extranjera y los cambios de derechos de la propiedad, tiene que haber aumentado la dispersión de las productividades y por tanto la desigualdad en la distribución del ingreso" (16). La parte de este trabajo sobre México analiza la distribución del ingreso corriente monetario desde 1977 a 2008, y la del ingreso corriente total desde 1992 a 2008. ${ }^{7}$ Para Cortés y a partir de los datos que presenta -cuadro 1 y gráfica 2- concluye que el período analizado no ha habido modificaciones sustantivas. En consecuencia:

7 La diferencia entre ambos conceptos de ingreso radica en que el segundo agrega al ingreso monetario el no monetario, que incluye la estimación del valor de la renta de las viviendas habitadas por sus propietarios, la valoración del autoconsumo, de los regalos recibidos y de los pagos en especie. 
se debería concluir que en los últimos tres quinquenios la sociedad mexicana no ha experimentado cambios en su estructura económica ni tampoco se ha modificado la distribución del poder entre las élites... la información muestra que ha habido una disminución significativa en la desigualdad del ingreso monetario en los últimos tres lustros, mientras que la distribución del ingreso total ha permanecido relativamente estable. Sobre la base del conocimiento disponible, ... a partir de los estudios de Enrique Hernández Laos ya citados, y el de Agustín Salvia (2010) sobre Argentina, se debería concluir que no ha habido modificaciones sustantivas en la dispersión de las productividades laborales por lo que, en principio, la información estaría registrando las variaciones de corto plazo a que aluden Przeworski y Wallerstein (2011-18-19).

Como también lo plantearon Esquivel, Lustig y Scott (2010), para Cortés la desigualdad en los ingresos monetarios en sensible a la participación relativa de los hogares en el decil más rico:

Las reducciones en la desigualdad en ambos tipos de ingresos registradas en 1984, 1996 y 2000-2004 se deben esencialmente a caída en la participación relativa del décimo decil; los datos de 1984 reflejan la distribución de los ingresos después de la crisis del petróleo de 1982; los de 1996 los ingresos de los hogares a casi dos años de la contracción económica de 1994-1995 y el de 2002 corresponde al estancamiento vivido por México entre 2000 y 2002-2004 (2011: 19).

Conviene subrayar lo que enfatiza Cortés respecto a quiénes son los actores que componen ese décimo decil cuya caída de ingresos juega un papel crucial en explicar la reducción de la desigualdad por ingresos:

estudios previos han mostrado que en este estrato se encuentran los hogares de la clase media alta, su ingreso lo obtienen por su desempeño como empresarios o como asalariados no agropecuarios. Incluye profesionistas, ya sea que trabajen por cuenta propia o como asalariados; técnicos, trabajadores de la educación y artistas; funcionarios públicos o funcionarios privados; oficinistas y comerciantes; vendedores y agentes de ventas. (Cortés y Rubalcava, 1991: 59-83 y Cortés, 2000: 57-75) (2011:20)

Así una explicación a las caídas de la desigualdad después de las crisis económicas:

se deberían a las reducciones en los sueldos y salarios reales de los burócratas así como de otros sectores (maestros y empleados universitarios) cuyos salarios son determinados institucionalmente y no por el mercado (Rubalcava, 1998: 97-98 y 128-139). Las políticas de austeridad económica que han aplicado los gobiernos mexicanos para enfrentar las crisis recurrentes se reflejan principalmente en la participación relativa del décimo decil. Las variaciones en la desigualdad en la distribución del ingreso observadas desde la década de los noventa en adelante no estarían reflejando cambios en la heterogeneidad estructural ni una intención gubernamental por reducir la desigualdad, sino la reacción del gobierno para estabilizar sus gastos. En épocas normales los ingresos de estos sectores se recuperan y retorna la inequidad económica (2011-20-21). 
En cualquiera de las dos interpretaciones sobre la evolución de la desigualdad en México: una que combina efectos en el mercado laboral de mano de obra cualificada versus a la no cualificada con la migración -a través de las remesas- y con la política pública de transferencias monetarias a los más pobres, y otra que enfatiza sobre todo el comportamiento del décimo decil -los más ricos- en la reducción de la desigualdad; por distintas vías ambas interpretaciones nos dicen que la reducción de la desigualdad se ha dado en el contexto de una economía con exiguo crecimiento - o en un contexto de "estancamiento estabilizador".

Los argumentos de los distintos estudios recientes sobre la desigualdad en América Latina -pero con énfasis en México- convergen alrededor de dos grandes ejes para enfrentar la desigualdad: la necesidad de impulsar el crecimiento de la economía y la necesidad de armar al Estado en sus ámbitos redistributivos.

Las características múltiples de la desigualdad así como sus mecanismos de trasmisión deben poner en guardia a quienes propongan fórmulas simplistas para enfrentarla. Desde que se necesita una educación más universal en todos los grados y de mejor calidad, al impulso de la infraestructura, a una cobertura universal en salud o mecanismos de protección social como seguro contra el desempleo o sistemas más amplios de pensiones; en todos los casos estamos implicando el imperativo del crecimiento económico y de la intervención del Estado en los distintos mercados.

De suerte que todos los estudios de organismos multilaterales o de especialistas en lo individual desembocan en la discusión sobre la naturaleza del Estado en esta coyuntura que intenta separarse -todavía sin fortuna- de los rasgos más groseros del neoliberalismo en su negativa a reconocer algún papel positivo del Estado como promotor del desarrollo.

Por ello son hoy aún más útiles las reflexiones que Cordera hacía en los noventas:

Asumir la redefinición nacional que impone el cambio del mundo no lleva a la negación del papel del Estado sino más bien, a su reforzamiento cualitativo. Este es el eje de la reforma del Estado. El tema y el problema no es cuánto Estado sino qué Estado... De lo que se trata ahora es de remover los obstáculos arcaicos al funcionamiento de los mercados y consecuentemente de pensar -como proyecto y perspectiva- los términos de una economía pública no necesariamente enclavada en una economía estatizada y ni siquiera dentro de una economía con una fuerte intervención estatal directa; se buscaría más bien erigir un conjunto de mecanismos de regulación desde el Estado y desde los diferentes estratos organizados de la sociedad (1993:302-203).

A lo anterior habría que añadir la argumentación del grupo de economistas que elaboraron casi veinte años después el documento sobre el "Nuevo curso", y que asume los errores que en los noventas se cometieron en torno a la sincronización de las reformas:

Es importante reflexionar sobre la secuencia que deben tener las reformas, sin perder de vista su necesaria articulación, pues de lo contrario se estaría incurriendo nuevamente en el error de plantear reformas inconexas y soluciones parciales. Resulta indudable que el puente entre las políticas macroeconómicas para aumentar la capacidad de respuesta de la economía mexicana y el resto de las reformas estructurales es la reforma fiscal, pero aprobar ésta sin llevar 
a cabo reformas sociales y laborales impedirá superar algunos de los problemas más graves que enfrenta nuestra economía, como el estancamiento del empleo formal y la insuficiencia y el deterioro de los sistemas públicos de salud y educación.

El propósito de ese esfuerzo reformador sólo se puede entender en la perspectiva de:

asumir la emergencia económica y social [que] constituye el punto de partida para el diseño de una estrategia destinada a responder a un objetivo central cuya realización plena debe ubicarse en el mediano y largo plazos, pero cuyo cumplimiento debe empezar a materializarse de inmediato: el establecimiento de un sistema de bienestar basado en derechos económicos y sociales exigibles, de alcance universal, y especialmente orientado a la promoción del bienestar juvenil, sin descuidar la atención de otros grupos vulnerables de la sociedad, también directamente afectados por la crisis y sus secuelas (2009:45).

Es la reforma fiscal empero, la que adquiere un lugar clave en cualquier reforma económica que aspire a romper el estancamiento económico. Los autores del "Nuevo curso" -entre los que se cuenta el propio Cordera- argumentan que:

la decisión de utilizar la política fiscal para la recuperación aún enfrenta resistencias, pues hay sectores conservadores que insisten que más que ampliar el gasto, la estrategia debería pasar por la reducción de impuestos. No obstante, la evidencia muestra que ese tipo de medidas, aplicadas por la administración republicana de Estados Unidos entre 2001 y 2008 , no hizo sino incrementar el desequilibrio del sector público sin contribuir a un aumento de la inversión productiva. Conviene reconocer, sobre todo, que la disputa ideológica sobre las políticas económicas dejó de circunscribirse al ámbito académico y es ya un debate político, lo que implica que las políticas económicas tienden a ser objeto de una abierta deliberación democrática, lo que no necesariamente ha ocurrido en la historia previa de la definición de las estrategias de desarrollo en los países latinoamericanos y, en particular, en el caso de México.

Más aún, añaden que "la necesidad de una profunda reforma fiscal no implica solamente avanzar hacia una de las múltiples reformas que necesita el país. Por el contrario, es el eje de la transformación social, de la articulación de un nuevo arreglo social que combine ampliación del bienestar para el conjunto de la población priorizando la condición de las grandes mayorías desfavorecidas, ampliación de las capacidades productivas de la economía y fortalecimiento de la convivencia armónica en el marco de la democracia participativa y el Estado de derecho."

Visto desde el lado del crecimiento de la economía el pensamiento más contemporáneo en general está a una enorme distancia de la propuesta de Kuznets que argumentaba que la distribución del ingreso tendría que comenzar siendo muy desigual para impulsar el crecimiento y luego tendería a igualarse conforme se diera convergencia de productividades. Precisamente la característica señalada por varios economistas ligados a CEPAL en los setentas y luego por Pinto y Fajnzylber respecto a la heterogeneidad estructural hace difícil que eso ocurra de manera "automática" sin intervenciones públicas. Más aun todos los estudios de organismos multilaterales, revisados en este documento, subraya el papel 
dañino que juega en sí misma la desigualdad en el diseño de instituciones y de políticas públicas.

El tipo de Estado que se aspira alcanzar tiene necesariamente que hacerse cargo de las deformaciones que emerge de las reformas estructurales de los noventa. Levy y Walton (2009) proponen una interpretación desde la economía política basada en que:

las desigualdades enquistadas -literalmente desigualdades atrincheradas- sostenidas a través de un equilibrio político que comparte rentas -rent-sharing politicial equilibrium-, es la fuente principal de las ineficiencias y del débil crecimiento. Más aún, este equilibrio ha sido resiliente a la democratización en formas tales que pueden ser explicadas por la naturaleza de las fuerzas subyacentes" (Traducido directamente del inglés, 2009-1).

Su interpretación no difiere sustancialmente de varias interpretaciones de los críticos a las políticas públicas de los fundamentalistas del mercado. ${ }^{8}$

Las interpretaciones de los llamados "reformadores del mercado" se sustentan en la idea central que "es en el carácter incompleto de las reformas económicas donde reside la mayor explicación del bajo crecimiento económico en México" (2009:5). Manejan la ya vieja tesis de que es mejor un "Estado regulador" que un "Estado productor".

Por su lado, según Levy y Walton los críticos de los reformadores del mercado argumentan que más allá de la secuencia y en la implementación de las reformas, los errores sistémicos son producto de una agenda de reformas conceptualmente equivocada. Subrayan además que muchas de las reformas se implementaron -por ejemplo las privatizaciones en el sector bancario, en telecomunicaciones o en infraestructura carretera- antes que se hubiera creado un marco regulatorio apropiado. Así afirman que:

los procesos de desregulación y privatización no han significado la transición de un estado productor a un estado regulador. Más bien ha sido un progresivo desmantelamiento del estado que ha sido incapaz, y quizás reacio, a defender a las pequeñas y medianas empresas y a los consumidores frente a las capturas de rentas de unas cuantas y enriquecidos grupos privados" (Traducida directamente del inglés, 2009:10).

8 Ver sobre todo a Luis Salazar (Salazar, Luis, "Agotamiento de la hegemonía revolucionaria y transición política", en México a fines de siglo Tomo II, compiladores: Blanco, José Joaquín y Woldenberg, José, Fondo de cultura económica, México, 1993), Rolando Cordera (Cordera, Rolando, "Desarrollo y crisis de la economía mexicana", FCE, México, 1981; Cordera, Rolando, "Los límites del Reformismo: la crisis del capitalismo mexicano", Cuadernos Políticos, Ed. Era, Núm., 2, $\stackrel{\sim}{\sim}$ México, 1974), Nora Lustig (Lustig, Nora, "México: the remaking of an economy", Brookings, USA, 1992) para México; y Stiglitz (Stiglitz, J. A., "More instruments and broader goals: Moving toward the post-Washington consensus, 1998" Wider Annual Lecture, Helsinki, Universidad de las Naciones Unidas, World Institue for Development Economics Research, 1998) y Rodrik (Rodrik, Dani, "The new global economy and developing countries: making openness work", Overseas Development Council, USA, 1999.) y los propios reportes del Banco Mundial (Banco Mundial, "Desigualdad en América Latina y el Caribe: ¿Rompiendo con la Historia?", BM, Washington, D.C. USA, 2005; Ferranti De, David, Guillermo E. Perry, Francisco H. G. Ferreira y Michael Walton, "Desigualdad en América Latina y el Caribe. ¿Ruptura con la historia?", Banco Mundial, Washington DC, 2004). 
En síntesis los críticos de las reformas de mercado, según Levy y Walton argumentan que

un crecimiento [económico] rápido no puede ocurrir en el contexto de un estado que carece de credibilidad en proveer mecanismos institucionales para un aprovisionamiento social eficiente y equitativo y para una regulación efectiva de los oligopolistas en una economía integrada en los mercados globales" (2009:11).

Desde la perspectiva de los autores del ensayo introductorio de ese libro colectivo ${ }^{9}$ el equilibrio político en México "está caracterizado por un alto grado de búsqueda de rentas -rent-seeking- que en un contexto de alta desigualdad continua, se traduce en comportamientos que reducen la eficiencia y concentran la riqueza"; es decir, "el problema no se deriva del adjetivo puesto al estado -productor, regulador o distributivo- sino con las instituciones que configuran los comportamientos del estado, es decir, sus incentivos, sus reglas que gobiernan el funcionamiento día a día y su rendición de cuentas"(2009:14). En esas condiciones subrayan los autores:

una nomenklatura de todos los partidos políticos, baraja y equilibra los intereses de aquellos que desde los negocios privados y la fuerza de trabajo organizada tienen capacidad para ejercer la voz y el poder cuando son necesarios para mantener y reproducir su poder, mientras que la mayor parte de la población sólo es capaz de ejercer su derecho al voto en las elecciones programadas" (ibid::14).

La interpretación que proponen Levy y Walton sobre los problemas del magro crecimiento económico en México está basada en el marco conceptual que proponen varios economistas citados por ellos y que se resume en el World Development Review 2006 (2005) sobre equidad y desarrollo. Este texto parte de dos tipos de influencias entre crecimiento y equidad: "interacciones entre las imperfecciones del mercado y la distribución de riqueza, poder y estatus... y los efectos de la desigualdad en el poder y la influencia en la selección de políticas y en el diseño de instituciones..." (2009:16).

En breve, un proyecto reformador para el México cuyo centro sea el combate a la desigualdad requiere replantearse el estilo de desarrollo tomando a la reforma fiscal como la reforma articuladora, rescatando las políticas sectoriales y los mecanismos de financiamiento estatal, impulsando el desarrollo regional a partir de privilegiar los encadenamientos productivos y negociando una inserción soberna en los procesos de globalización que hoy se encuentran fuertemente cuestionados por las crisis económicas mismas. El propósito central de este replanteamiento es reducir la pobreza y la desigualdad sobre la base de colocar en el centro del nuevo curso de desarrollo, el imperativo de crecimiento económico y la garantía de derechos sociales exigibles. Pero todo lo anterior exige fortalecer a un estado de suyo debilitado no buscando una imposible e indeseable reedición del pasado, sino justamente por el contrario asumiendo la necesidad de pasar de un Estado patrimonialista y clientelar a un Estado de la sociedad, es decir un Estado social de dere-

9 El ensayo de Levy y Walton se intitula Equidad, competencia y crecimiento en México y el libro colectivo ¿No hay crecimiento sin equidad? (2009). 
cho. Cordera enumere diez desafíos que la desigualdad en México plantea, de entre los cuales quisiera destacar los siguientes:

- No puede haber poderío exportador sin un mercado interno robusto. Y no hay mercado interno amplio y dinámico sin cambios en la estructura distributiva a favor de la equidad y sin un crecimiento alto y sostenido del producto y del empleo...

- La democracia resiente la desigualdad porque ésta pone en entredicho su discurso sustantivo, que es igualitario en forma y fondo...

- Así vista, la desigualdad desafía directamente al mercado realmente existente que se pone a su servicio y reproducción. De aquí la necesidad de una reforma institucional que profundice y amplíe la competencia, y contribuya a recrear los mecanismos estatales de mediación del conflicto social y de producción sistemática de instrumentos de seguridad colectiva universal...[Para ello] es indispensable empezar por las políticas que dinamicen el mercado a través de la inversión...

- De aquí la pertinencia de una tercera reforma que emane del reconocimiento intenso de la sociedad desigual que es México. Más que en la economía política, donde hay que hurgar es en la matriz de valores que articula las prácticas de la política del poder y las convicciones de la economía... De esta reforma, que en otra parte he bautizado como una reforma intelectual y moral orientada a hacer del Estado un verdadero Estado social, pueden surgir nuevas formas de cohesión y articulación sociales...

- Una convocatoria para "socializar" al Estado, como vía para su renovación y para darle sustrato institucional al compromiso de la sociedad con la igualdad, puede llevar a... un compromiso congruente de las élites con la búsqueda de la equidad...

- No hay globalización exitosa ni democracia madura sin la existencia y reproducción de capacidades nacionales de adaptación al progreso técnico universal; sin creatividad e innovación productiva vinculadas a la educación superior y la investigación científica (2006:64-68).

\section{Bibliografía}

Acemoglu, D., S. Johnson y J. A. Robinson, "Reversal of Fortune: Geography and Institutions in the Making of the Modern World Income Distribution", Quaterly journal of Economics, 117, November 2002.

Acemoglu, D., S. Johnson y J. A. Robinson, "The Colonial Origins of Comparative Development: An Empirical Investigation", The American Economic Review, Vol. 91, No. 5, December, 2001.

Birsdall, N., A. de la Torre y F. Valencia, "The Washington Consensus: Assessing a damaged brand", Working Paper 213, Center for Global Development, Washington, DC, 2010.

Birsdall, N., y A. de la torre, "Washington Contentious. Economic Policies for Social Equity in Latin America. Findings of the Commission on Economic Reform in Unequal Latin America Societies" sponsored by the Carnegie Endowment for International Peace and the Inter-American Dialogue, 2001. 
CEPAL, "La hora de la igualdad. Brechas por cerrar, caminos por abrir.", LC/G.2432(SES.33/3), Santiago de Chile, 31 de Mayo de 2010. http://www.eclac.org/publicaciones/xml/0/39710/100604_2010114-SES.33-3_La_hora_de_la_igualdad_doc_completo.pdf

Cordera, Rolando, "La desigualdad marca nuestra historia" en Toledo F., Enrique Florescano y José Woldenberg (coord.), Los desafíos del presente mexicano, Taurus, 2006.

Cordera, Rolando y Leonardo Lomeli, "El papel de las ideas y las políticas en el cambio estructural, 19822004" en Rolando Cordera y Carlos Javier Cabrera (coordinadores), El papel de las ideas y las políticas en el cambio estructural en México, Fondo de Cultura Económica, (Lecturas de El Trimestre Económico 99), México, 2008.

Cortés, Fernando, "Desigualdad económica y poder", documento inédito, 2011.

Cortés F. (2008), Procesos sociales y evolución de la distribución del ingreso monetario (1997-2004), en Rolando Cordera y Carlos Javier Adame (coords.), El papel de las ideas y las políticas en el cambio estructural de México. México, El Trimestre Económico (Lecturas), pp. 415-440.

Cortés F., La distribución del ingreso en México en épocas de estabilización y reforma económica, CIESAS y Miguel Ángel Porrúa, México, 2000.

Cortés F. y R. M. Rubalcava, Autoexplotación forzada y equidad por empobrecimiento: La distribución del ingreso familiar en México, 1977-1984. El Colegio de México, México, 1991.

Engerman, Stanley L., and Kenneth L. Sokoloff., "Factor Endowments, Institutions, and Differential Paths of Growth Among New World Economies: A View from Economic Historians of the United States", In Stephen Haber, ed., How Latin America Fell Behind, Stanford University Press, Stanford, Calif., 1997.

Esquivel, G. "Piloto automático procíclico: El diseño y conducción de la política macroeconómica en

México", documento preparado para el Seminario La Economía Mexicana en el 2010, El Colegio de México, México, 2008.

Esquivel, Gerardo, Nora Lustig, adn John Scott, "Mexico: A decade of falling Inequality: Market Forces or State Action?", en Luis Felipe López-Calva and Nora Lustig, eds., Declining Inequality in Latin America: A Decade of progress?, Brokings Institution Press and United Nations Development Programme, 2010.

Fajnzylber, F., La industrialización trunca de América Latina, Editorial Nueva Imagen, México, D.F., 1983.

Fajnzylber F. "Industrialización en América Latina: De la caja negra al casillero vacio", Cuadernos de la CEPAL 60, Naciones Unidas, Santiago de Chile, 1990.

Ferrantis De, David, Guillermo E. Perry, Francisco H. G. Ferreira y Michael Walton, Desigualdad en América Latina y el Caribe. ¿Ruptura con la historia?, Banco Mundial, Washington DC, 2004.

Genicot, G. and D. Ray, "Aspirations, Inequality, Investment and Mobility", Georgetown University and New York University, mimeo, 2009.

Guerrero, I., L. F. López-Calva y M. Walton, "The inequality trap and its to low growth in Mexico", en Santiago Levy y Michael Walton (eds.), No Growth without Equity? Inequality, Interests, a nd competition in Mexico, Banco Mundial y Palgrave, Macmillan, Washington, D. C., USA, 2009.

Latinobarómetro, 2004. (Ver: http://www.jdsystems.es/latinobarometro/LATBD_Latinobarometro_ Informe_2004.pdf).

Latinobarómetro, 2010. (Ver: http://www.jdsystems.es/latinobarometro/LATBD_INFORME\%20 LATINOBAROMETRO\%202010.pdf).

Levy, S., Good intentions, bad outcomes: social policy, informality, and economic growth in Mexico, Brookings Institution Press, 2008.

Levy S. y M. Walton (2009), "Equity, Competition, and Growth in México: An overview, en Levy Santiago 
and Michael Walton (Eds) No Growth Without Equity? Inequality,Interests and Competition in Mexico, Palgrave Macmillan and The World Bank,Washington DC.

Lewis, W. A. (1973): "Desarrollo económico con oferta ilimitada de mano de obra" en A. N. Agarwala y S.

P. Singh (comp.), La economía del subdesarrollo, Madrid, Tecnos, pp. 333-374.

Lewis, W. A. "Desarrollo económico con oferta ilimitada de fuerza de trabajo", El Trimestre Económico 26 (108), octubre.

Li H., L. Squire, H. Zou, " Explaining international and inter-temporal variations in income inequality, The economic journal, núm. 108, págs. 1 a 18.

López-Calva, L. F. y N. Lustig (Eds.), "Declining Income Inequality in America: A Decade of Progress?", Brookings Institution Press, Baltimore, MD, 2010.

López-Calva, Luis Felipe, and Nora Lustig, eds. Declining Inequality in Latin America: A Decade of progress?, Brokings Institution Press and United Nations Development Programme, 2010.

Nebel, M. y P. Flores-Crespo (Eds.), Desarrollo como libertad en América Latina. Fundamentos y aplicaciones, Universidad Iberoamericana, Ciudad de México, México, 2010.

OHCHR (Oficina del Alto Comisionado para los Derechos Humanos), "Preguntas frecuentes sobre el enfoque de derechos humanos en la cooperación para el desarrollo", Oficina del Alto Comisionado de las Naciones Unidas, 2006

Pagés, C., G. Pierre, and S. Scarpetta. Job Creation in Latin America and the Caribbean: Recent Trends and Policy Challenges, Palgrave MacMillan and the World Bank, Washington, DC, 2009.

Piketty, T. (2005), "Social Mobility and Redistributive Politics" ,Quaterly Journal of Economics,núm. 111, págs. 1004 a 1042.

Piketty, T. (2007) Top incomes over the Twentieth century: a summary of main findings, en Anthony Atkinson y Thomas Piketty (eds.), Top Incomes over the 20th Century: A Contrast Between Continental European and English-Speaking Countries. Oxford, OxfordUniversity Press, pp. 1-117.

Piketty, T., "Social Mobility and Redistributive Politics", Quaterly journal of Economics, núm. 111, 1995.

Pinto, Anibal, Heterogeneidad estructural y modelo de desarrollo reciente de la América Latina en Inflación: raíces estructurales, México, D.F.: Fondo de Cultura Económica, 1973.

PNUD, "Informe Regional sobre Desarrollo Humano para América Latina y el Caribe 2010: Actuar sobre el futuro: romper la trasmisión inter-generacional de la desigualdad", Programa de las Naciones Unidas para el Desarrollo, Primera Edición, San José, Costa Rica, 2010.

PNUD, "La democracia en América Latina: Hacia una democracia de ciudadanas y ciudadanos", Programa de las naciones unidas para el desarrollo, Buenos Aires, Argentina, Abril de 2004.

Prebisch, R. (1949), El desarrollo económico de la América Latina y algunos de sus principalesproblemas (E.CN.12/89), Naciones Unidas, Santiago de Chile.

Prebisch, Raúl, "El desarrollo económico de la América Latina y algunos de sus principales problemas", v. 1, p. 63-129. En: NU. CEPAL. Cincuenta años del pensamiento de la CEPAL: textos seleccionados. Santiago: Fondo de Cultura Económica/CEPAL, 1998. 2 v.

Przeworski, A. y M. Wallerstein, "Structural Dependence of the State on Capital", American Political Science Review, núm. 82., 1988.

Przeworski A. (2010), Qué esperar de la democracia: límites y posibilidades del autogobierno, Siglo XXI, Buenos Aires.

Rostow W. (1960), Las etapas del crecimiento económico: un manifiesto no comunista, Fondode Cultura Económica, México.

Scott, John, 2009 "Gasto Público y Desarrollo Humano en México: Análisis de Incidencia y Equidad", 
Working Paper for Informe de Desarrollo Humano de México 2008/2009, PNUD. Poverty Reduction Discussion Paper, México, 2009a.

"Redistributive Constraints under High Inequality: The Case of Mexico", document prepared for the UNDP project Markets, the State and the Dynamics of Inequality: How to Advance Inclusive Growth, coordinated by Luis Felipe López-Calva and Nora Lustig, 2009b. Available at www.undp.org/ latinamerica/inequality.

"The Incidence of Agricultural Subsidies in Mexico", paper for project on Agricultural Trade Adjustment and Rural Poverty: Transparency, Accountability and Compensatory Programs in Mexico, Woodrow Wilson International Center for Scholars, Mexico Institute, 2009c.

Sen. A. K., Commodities and Capabilities, Paises Bajos, Amsterdam, 1985.

Sen. A. K., "Democracy as a Universal Value", Journal of Democracy, 10(3): 3-17., 1999.

Sen. A. K., Development as Freedom, Oxford University Press, Oxford, 1998.

Sen. A. K., Equality of what? en McMurrin, S. (ed.) The Tanner Lectures on Human Values, Vol.1. Cambridge: Cambridge University Press, Cambridge, 1980.

Sen. A. K., "The Three R's of Reform", Economic and Political Weekly, May 7, 2005.

Sokoloff, Kenneth L., and S. L. Engerman, "History Lessons: Institutions, Factors Endowments, and Paths of Development in the New World", The Journal of Economic Perspectives, Vol. 14, No. 3, Summer, 2000.

Sundrum, R.M (1990). Income Distribution in Less Developed Countries. Londres, Routledge.

Tokman, Victor, 1979, Dinámica del trabajo urbano: el sector informal en América Latin en Rubén Katzman y José Luis Reyna, compiladores, Fuerza de trabajo y movimientos laborales en América Latina. El Colegio de México, 1979.

Tokman V. ( 1979), " Dinámica del trabajo humano: el sector informal en América Latina", en Ruben Katzman y José Luis Reyna (comps.) Fuerza de trabajo y movimientos laborales en América Latina, El Colegio de México, México.

Toledo F., Enrique Florescano y José Woldenberg coord., Los desafíos del presente mexicano, Taurus,2006.

Vuskoviç P. (1993), Pobreza y desigualdad en América Latina, Centro de Investigaciones Interdisciplinarias, UNAM, México.

Yasukawa, Y., Ortuño y Brockman, "Derechos y Política Social", publicado en la revista Proceso del periódico La Prensa de La Paz, Bolivia, 28 de diciembre de 2009. 